March 26, 1995

LBL-36915

\title{
Quantum Mechanical Coherence, Resonance, and Mind.*
}

\author{
Henry P. Stapp \\ Theoretical Physics Group \\ Lawrence Berkeley Laboratory \\ University of California \\ Berkeley, California 94720
}

Invited Contribution to the Norbert Wiener Centenary Congress, held at Michigan State University, Nov. 27 - Dec. 3, 1994.

\begin{abstract}
Norbert Wiener and J.B.S. Haldane suggested during the early thirties that the profound changes in our conception of matter entailed by quantum theory opens the way for our thoughts, and other experiential or mind-like qualities, to play a role in nature that is causally interactive and effective, rather than purely epiphenomenal, as required by classical mechanics. The mathematical basis of this suggestion is described here, and it is then shown how, by giving mind this efficacious role in natural process, the classical character of our perceptions of the quantum universe can be seen to be a consequence of evolutionary pressures for the survival of the species.
\end{abstract}

${ }^{*}$ This work was supported by the Director, Office of Energy Research, Office of High Energy and Nuclear Physics, Division of High Energy Physics of the U.S. Department of Energy under Contract DE-AC03-76SF00098. 
.

.

Q9 reycled pape 


\section{DISCLAIMER}

Portions of this document may be illegible in electronic image products. Images are produced from the best available original document. 


\section{Introduction}

This session of the congress is entitled "Leibniz, Haldane, and Wiener on Mind". Accordingly, my talk will deal with issues that are often considered to be more philosophical than mathematical. However, the logical basis of my remarks is the Hilbert space formalism of quantum mechanics.

I introduce the subject by giving some quotations from Haldane's article "Quantum mechanics as a Basis for Philosophy"1, from Wiener's article "The Role of the Observer", ${ }^{2}$ and from Bohm's Commentary ${ }^{3}$ in the Collected Works of Norbert Wiener.

\section{Haldane:}

1. Biologists have as yet taken but little cognizance of the revolution in human thought which has been inaugurated by physicists in the last five years, and philosophers have stressed its negative rather than its positive side.

2. If mind is to be regarded as expressive of the wholeness of the body, or even of the brain, it should probably be thought of as a resonance phenomenon, in fact part of the wave-like aspect of things.

3. If mind is a resonance phenomenon it is at once clear why it cannot be definitely located, either in space or time, though it is obviously enough connected with definite events in a definite material structure.

4. But it is, I think, of importance that philosophers, and even ordinary persons, should realize that a thorough-going materialism is compatible with the view that mind has many of the essential properties attributed to it by metaphysicians. The theory here presented does not reduce it to an epiphenomenon of matter, but exhibits it as a reality interacting with ordinary material systems.

5. It has been my object to suggest that the progress of modern physics has made such a unified view more readily attainable than appeared likely ten years ago. 


\section{Wiener:}

1. The Platonist believes in a world of essence, of cleanly defined ideas and cleanly defined propositions concerning these ideas, into which we may enter as spectators, but never as participants. They are out of time, and time is irrelevant to them.

This is pure dogma, and does not check with what we should naively expect. Of course, our experiences must have some reference outside themselves, in the sense that they cannot be considered as completely closed and isolated. Otherwise there could be no knowledge at all. This by no means asserts that the experience has a reference entirely unaltered by our participation.

2. Thus physics, the most exact of all sciences, has had to have a thorough logical housecleaning. We no longer conceive the laws of physics to apply to some mystical world of reality behind our observations and instruments: they merely constitute an intelligible statement of the manner in which our observations and the readings of our instruments hang together.

3. The philosophy of Hume furnishes the dreadful example of what happens to an empiricism which seeks its fundamental reality in the fugitive sensedata of immediate experience. If the raw stuff of our experience does not contain something of a universal nature, no manipulation can ever evoke anything which might even be mistaken for a universal.

4. Science is the explanation of process. It is neither possible under a rationalism, which does not recognize the reality of process, nor under an empiricism, which does not recognize the reality of explanation. 


\section{Bohm:}

1. In [36G] Wiener goes into the role of the observer, which has been emphasized in the quantum theory. He points out that in art, drama, psychology, and medicine we are all familiar with areas of experience in which the observer is not merely a passive receiver of perceptions but, on the contrary, plays an active and essential role in all that is seen.

Wiener proposes that in physics and mathematics a similar approach is now called for. We do not ask for a mystical world of reality behind our observations. Physics is a coherent way of describing the results of our observations and what is done with them.

2. .... in all his thinking Wiener has consistently and coherently sought to achieve what he already indicated in the earliest of his papers on the quantum theory, i.e. something that "possesses more of an intrinsic logical necessity" than is possessed by already existing modes of thoughts.

These quotations highlight the fact that the discovery of quantum mechanics has opened up the possibility that mind - - - i.e., the realm of experiential things, such as our thoughts, ideas, and perceptions - - - may not be epiphenomenal after all: mind may be something quite different from the causally inert by-product of the microscopically specified and determined mechanical processes in our brains (or bodies) that the principles of classical mechanics require it to be.

This possibility that mind is an interactive and dynamically efficacious aspect of nature, not reducible to the locally determined mechanical features that characterize the "matter" of classical physics, arises from the circumstance that quantum dynamics has an element of wholeness that is not reducible to those local aspects of nature, but that rather complements them, and interacts with them. This added element is directly tied to our thoughts by the basic rules of quantum mechanics.

During the twenties and thirties our detailed scientific understanding of brain processes, and their connection to our thoughts, was too rudimentary to allow this possibility offered by quantum mechanics to be related to empirical findings. Now, however, we are entering a period of intensive empirical scruting 
of brain processes and their connections to thoughts. In this new climate the fact that quantum mechanics provides a scientifically based mathematical setting that allows mind to complement and interact with the aspect of nature that characterizes the "matter" of classical mechanics has become the basis of a line of research that is being aggressively pursued. This paper presents some recent results in this area, but begins by describing the situation as it was understood in the thirties.

The most orthodox interpretation of quantum theory is the one formulated by Niels Bohr. It was radical in its time because it rejected the prevailing idea that the ultimate task of science was to develop a mathematical model of the universe. Quantum philosophy asserted that the proper task of science was merely to formulate rules that allow us to calculate all of the verifiable relationships among our experiences. According to Bohr:

"In our description of nature the purpose is not to disclose the real essence of phenomena but only to track down as far as possible relations between the multifold aspects of our experience." 4

and

"Strictly speaking, the mathematical formalism of quantum mechanics merely offers rules of calculation for the deduction of expectations about observations obtained under well defined experimental conditions specified by classical physical concepts." 5

The format for using quantum theory is as follows:

Let $A$ be a classical description of an experimental set up.

Let $\mathrm{B}$ be a classical description of a possible outcome of this experiment.

By a "classical description" Bohr means a description in terms of ordinary language, elaborated by the concepts of classical physics. It is a description of what the technicians who set up the experiment should do, and what the observers who observe the results of the experiment might see, or otherwise experience.

A mapping from these "classical descriptions" to quantum operators is defined, essentially by calibrations of the devices: 


$$
\begin{aligned}
& A \Longrightarrow \rho_{A} \\
& B \Longrightarrow P_{B} .
\end{aligned}
$$

Here $\rho_{A}$ is the density operator (or statistical operator) that corresponds to the classical description $A$, and $P_{B}$ is the projection operator (i.e., $P_{B}^{2}=P_{B}$ ) that is the Hilbert space representation of the outcome specified by the classical description $B$. The basic quantum postulate is that the probability $P(B ; A)$ that an outcome that satisfies the specifications $B$ will occur under conditions that satisfy the specifications $\mathrm{A}$ is given by

$$
P(B ; A)=\operatorname{Tr} P_{B} \rho_{A},
$$

where $\operatorname{Tr}$ is the trace operator in Hilbert space:

$$
\operatorname{Tr} X=\sum_{i}<i|X| i>
$$

Here the index $i$ labels the vectors of a complete orthonormal basis:

$$
<i \mid j>=\delta_{i j}= \begin{cases}1 & \text { for } i=j \\ 0 & \text { for } i \neq j\end{cases}
$$

and

$$
\sum_{i}|i><i|=I \text {. }
$$

The symbol $I$ repesents the identity operator defined by $I|X\rangle=|X\rangle$ for all $|X\rangle$. (If the detectors are not $100 \%$ efficient then the operator $P_{B}$ must be replaced by an efficiency operator, $e_{B}$, but I shall ignore here this possible complication, in order to focus on the central points.)

Notice that there is no mention here of any "collapse of the wave function" or "reduction of the wave packet" or "quantum jump". (See below.) Notice also that the formulation is pragmatic: it is a description of how to use the theory; and the basic realities in the description are the experiences of the human beings who set up experiments and observe their outcomes. The objectivity of the theory is secured by formulating the specifications on the preparations and observations in terms of the "objective" language of classical description: there is no greater dependence here on individual human beings than there is in classical physics. 
A principal feature of a classical description is that objects and properties are assigned to locations that are definite, at least at the level of our perceptions: the center of an observable "pointer" that indicates the outcome specified by a measuring device does not lie simultaneously at two locations that can be perceived to be different. The whole idea of a measurement, or of an experiment, refers here to things that can be perceived.

Einstein, and many other scientists, objected to this introduction of human observers into the formulation of the basic physical theory. According to Einstein:

"Physics is an attempt to conceptually grasp reality as it is thought independently of its being observed." 6

and

"It is my opinion that contemporary quantum mechanics constitutes an optimum formulation of [certain] connections but forms no useful point of departure for future developments."

As regards "future developments" one may mention biological systems. Quantum theoretical ideas are important in describing and understanding the properties of the tissues of biological systems. However, living systems cannot be isolated from their environment. Yet the orthodox formulation of quantum theory demands that the observed system, which is the one represented in Hilbert space, be isolated from the observing system, which consists of the observers, their devices, and all systems coupled to them, between the time of preparation of the observed system and the time of its observation. Bohr himself stressed ${ }^{8}$ that this idealization cannot be achieved for biological systems, and that the scope of quantum theory, as he interpreted it, was correspondingly limited.

This isolation requirement fails also for cosmological system, because in this context the observers are inside the quantum system that is the object of study, and hence cannot be isolated from it.

John von Neumann, in his book" "Mathematical Foundations of Quantum Mechanics" examined the measurement problem, which is precisely the problem of specifying the connection between an observed system and the observing one. He started from the assumption, quite contrary to that of Bohr, that the entire system of observed and observer should be treated within the quantum 
formalism. His main result is easy to state.

Suppose we have a sequence of systems such that the first system is some atomic system that might be in a state $\psi_{11}$; that might be in a state $\psi_{12}$; or that might be in a superposed state $a \psi_{11}+b \psi_{12}$, with $|a|^{2}+|b|^{2}=1$. Suppose the second system is a measuring device that measures whether the first system is in state $\psi_{11}$ or $\psi_{12}$, in the sense that if the first system is originally in state $\psi_{11}$ and the second system is originally in some state $\psi_{20}$ then the combined system, originally $\psi_{11} \otimes \psi_{20}$, will evolve, due to the interaction between the two systems, into a state $\psi_{11}^{\prime} \otimes \psi_{21}$; whereas if the first system is originally in the state $\psi_{12}$, instead of $\psi_{11}$, then the combined original system $\psi_{12} \otimes \psi_{20}$ will evolve into the state $\psi_{12}^{\prime} \otimes \psi_{22}$, where $\psi_{22}$ represents a state of the device that is perceptually different from the state represented by $\psi_{21}$, so that an observer, by seeing whether the second system (the measuring device) is in state $\psi_{21}$ or $\psi_{22}$, can unambiguously infer whether the atomic system was originally in the state $\psi_{11}$ or $\psi_{12}$.

The linear nature of the law of evolution of the full quantum system consisting of the first and second systems ensures that if the first system had originally been in the state $\left(a \psi_{11}+b \psi_{12}\right)$, and the combined system had originally been in the state $\left(a \psi_{11}+b \psi_{12}\right) \otimes \psi_{20}$, then this original state would evolve into the state

$$
\psi=a \psi_{11}^{\prime} \otimes \psi_{21}+b \psi_{12}^{\prime} \otimes \psi_{22}
$$

But this state has a part corresponding to each of the two macroscopically distinguishable configurations of the device: e.g., it has a part, $\psi_{21}$, that corresponds, for example, to the pointer's having swung to the left, and it has a part, $\psi_{22}$, that corresponds, for example, to the pointer's having swung to the right. The general possibility (in principle) of exhibiting interference effects involving both terms of any superposition of states means that the two terms in the superposition $\psi$ must be combined as a conjunction (both parts must be present in nature) rather than as a disjunction (only one part or the other is present in nature). Yet only one or the other position of the pointer is ever observed, not both simultaneously. But then how does the "and" combination become transformed to an "or" combination?

To examine this problem von Neumann introduces a sequence of measuring devices, with each one set up to distinguish the two outcome states of the imme- 
diately preceding one in the sequence. The previous argument now generalizes: the original state

$$
\Psi_{10}=\psi_{11} \otimes \psi_{20} \otimes \psi_{30} \otimes \ldots \otimes \psi_{N 0}
$$

will evolve into

$$
\Psi_{1}=\psi_{11}^{\prime} \otimes \psi_{21} \otimes \psi_{31} \otimes \ldots \otimes \psi_{N 1},
$$

whereas the original state

$$
\Psi_{20}=\psi_{12} \otimes \psi_{20} \otimes \ldots \otimes \psi_{N 0} .
$$

will evolve into

$$
\Psi_{2}=\psi_{12}^{\prime} \otimes \psi_{22} \otimes \psi_{32} \otimes \ldots \otimes \psi_{N 2} .
$$

But then the linearity of the equation of motion ensures that the original state

$$
\Psi_{0}=\left(a \psi_{11}+b \psi_{12}\right) \otimes \psi_{20} \otimes \psi_{30} \otimes \ldots \otimes \psi_{N 0}
$$

will evolve into

$$
\begin{aligned}
\Psi & =a \psi_{11}^{\prime} \otimes \psi_{21} \otimes \psi_{31} \otimes \ldots \otimes \psi_{N 1} \\
& +b \psi_{12}^{\prime} \otimes \psi_{22} \otimes \psi_{32} \otimes \ldots \otimes \psi_{N 2}
\end{aligned}
$$

The wave functions $\psi_{N 1}$ and $\psi_{N 2}$ are taken to be the wave functions of the parts of the brain that are the brain correlates of the experiences of the human observer, in the two alternative possible cases defined by $\Psi_{1}$ and $\Psi_{2}$. Thus $\psi_{N 1}$ would represent the brain correlate of the experience of seeing a device outcome that indicates that the original state of the atom was $\psi_{11}$, whereas $\psi_{N 2}$ would represent the brain correlate of the experience of seeing a device outcome that indicates that the original state of the atom was $\psi_{12}$. But then if the original state of the atom were $\left(a \psi_{11}+b \psi_{12}\right)$, with $a \neq 0 \neq b$, the final state of the brain would have one component, $\psi_{N 1}$, that corresponds to the experience of seeing a device in a configuration that indicates that the original wave function of the atom was $\psi_{11}$, and another component, $\psi_{N 2}$, that corresponds to the experience of seeing a devices in a configuration that indicates that the original wave function of the atom was $\psi_{12}$. But how do we reconcile the fact that the final state $\Psi$ has two components corresponding to two different experiences, 
namely $\psi_{N 1}$, which corresponds to seeing a pointer swung to the left, and $\psi_{N 2}$, which corresponds to seeing that pointer swung to the right, with the empirical fact that only one or the other of the two possible experiences will actually occur? How has the "and" at the level of the device changed over to an "or" at the level of our experience.

The answer, if we apply the words of Bohr, arises from the assertion that "In fact, wave mechanics, just as the matrix theory, represents on this view a symbolic transcription of the problem of motion in classical mechanics adapted to the requirements of quantum theory and only to be interpreted by an explicit use of the quantum postulate." ${ }^{10}$. (Italics mine.)

The mathematical core of the quantum postulate is the probability rule (1.2):

$$
P(B ; A)=\operatorname{Tr} P_{B} \rho_{A} .
$$

In our example the projection operator $P_{B}$ associated with the observation of system $n(1 \leq n \leq N)$ in state $j(j=1$ or 2$)$ is (in Dirac's bra-ket notation)

$$
P_{n j}=\left|\psi_{n j}><\psi_{n j}\right| \times \prod_{s \neq n} I_{s}
$$

where $I_{s}$ is the unit or identity operator in the Hilbert space associated with system $s$. The $\left|\psi_{n j}\right\rangle$ are normalized so that

$$
<\psi_{n j} \mid \psi_{m \ell}>=\delta_{n m} \delta_{j \ell}
$$

The density operator for the final state, under the condition that the original state of the atom is $a\left|\psi_{11}>+b\right| \psi_{12}>$, is

$$
\rho_{A}=|\Psi><\Psi|
$$

where [(1.5f) transcribed into Dirac's notation]

$$
\begin{aligned}
\mid \Psi> & =a\left|\psi_{11}^{\prime}>\otimes\right| \psi_{21}>\otimes \ldots \otimes \mid \psi_{N 1}> \\
& +b\left|\psi_{12}^{\prime}>\otimes\right| \psi_{22}>\otimes \ldots \otimes \mid \psi_{N 2}>
\end{aligned}
$$

Then, in the case that the measurement outcome $B$ corresponds to finding the system $n(1 \leq n \leq N)$ in state $j(j=1$ or 2$)$, one obtains

$$
\begin{aligned}
P(B ; A) & =\operatorname{Tr} P_{n j} \rho_{A} \\
& =|a|^{2} \delta_{1 j}+|b|^{2} \delta_{2 j}
\end{aligned}
$$


That is, the probability of the outcome $j$ is either $|a|^{2}$ or $|b|^{2}$ according to whether the value of $j$ is 1 or 2 , and this result is independent of which of the $N$ possible systems is specified by $n$ : i.e., the probability for the outcome $j$ is independent of which one of the $N$ systems is considered to be the "measured" or "observed" one. Carrying the analysis up to the level of the brain correlate of the experience does not change the computed probability.

By combining the ideas of von Neumann and Bohr in this way we have resolved, in a certain sense, the measurement problem in a way that does not automatically exclude biological or cosmological systems. In this development the final system, system $N$, plays a special role: it provides the Hilbert space in which is represented of the immediate objects of our experiences. These experiences are the basis of Bohr's approach. However, Bohr did not recommend considering the brain correlate of the experience to be the directly experienced system, as, following the approach of von Neumann, has been done here.

According to the ideas of Bohr, the Hilbert-space state should not be considered to characterize the objectively existing external reality itself; it is merely a symbolic form that is to be used only to compute expectations that pertain to classically describable experiences. Each of the two states $\psi_{N 1}$ and $\psi_{N 2}$ is the brain correlate of a classically describable experience in which, for example, a "pointer" of an observable device is located at a well defined position. But a more general state such as $a \psi_{N 1}+b \psi_{N 2}$, with $a \neq 0 \neq b$, would evidently not be the brain correlate of any single classically describable experience. Hence its probability would not be something that it would be useful to compute: the "occurrence" of such an event would have no empirical meaning. The special role of classical concepts in the formalism therefore arises, according to this viewpoint, fundamentally from the circumstance that our perceptual experiences of the external world have, as a matter of empirical fact, aspects that can be described in classical terms.

In this Bohr-type way of viewing the theory the Hilbert space quantities are merely computational devices: the only accepted realities are the experiences of the observers. Thus the approach is fundamentally idealistic.

We can retain these basic experiential realities yet expand our mathematical representation of nature to include also a representation of the "physical" reality by adopting (with Heisenberg) the Aristotelian notion of "potentia": i.e., by 
conceiving the Hilbert-space state to be (or to faithfully represent) a reality that constitutes not the "actual" realities in nature, which are events, but merely the "potentia" for such actual events to occur. Then the Bohr-type experiential realities can be retained as the "actual" things of nature, while the Hilbertspace state becomes a representation of "objective tendencies" (in the words of Heisenberg) for such actual events to occur.

The notion that the real actual things in nature should occur only in conjunction with human brains is an idea that is too anthropocentric to be taken seriously. Indeed, Heisenberg proposes that actual events should occur already at the level of the first measuring device. However, as suggested already by our simple example, there is no empirical evidence to support the intuitively appealing notion that there are events at that purely mechanical level. That conclusion is the basic message that comes from the numerous detailed elaborations of von Neumann's analysis that have been carried out over the years: the simple example already exhibits the essential result.

In the present realistic approach the probability rule $P(B ; A)=\operatorname{Tr} P_{B} \rho_{A}$ is interpreted as the probability that an event corresponding to $B$ actually occurs under the condition that the state of the universe is specified by $\rho_{A}$.

If we were adhering to the pragmatic Bohr-type philosophy then it might be useful, for reasons of computational convenience, to push the level at which the event is supposed to occur down to a level such that any shift to a higher level will not change the computed probability significantly. But in a realistic context the placement of the actual events ought to be be governed by a general principle, not by reason of its practical convenience.

Putting aside, temporarily, this question of where to place any actual events that might occur outside the brain, let us focus on processes occurring inside human brains. Let us suppose, in line with our attempt to extend Bohr's pragmatic/idealistic interpretation to a realistic one, that the actual events in the brain occur only at the top level, i.e., at the level of the brain correlates of our conscious experiences; at the level of the states $\psi_{N 1}$ and $\psi_{N 2}$ of our earlier discussion. Then we arrive at the situation referred to by Haldane, Weiner, and Bohm. In this conception of nature we have, on the one hand, the "potentia", which is represented by the evolving Hilbert-space state. It constitutes the matter-like aspect of nature, in the sense that it is represented in terms of local 
quantities that normally evolve deterministically in accordance with local laws that are direct generalizations of the local laws of classical mechanics. But this is not the whole story. There are, on the other hand, also the "actual" events that we experience. These events are represented in Hilbert space by sudden changes in the state vector. These two aspects of nature are complementary: it makes no sense to have "tendencies" without having the events that these tendencies are the tendencies for; and it makes no sense to have separate experiential events with no reality connecting them. These two complementary aspects of nature interact: each actual event selects certain possibilities from among the ones generated by the evolving "potentia". Thus mind is no longer a causally inert epiphenomenon that can be reduced to the locally specified and determined matter-like aspects of nature: mind is rather an integral nonlocal aspect of reality that acts as a unit upon the local deterministic matter-like aspect of nature, which conditions this mental aspect but does not completely control it.

This completes my skeletal description of the mathematical basis of the idea of Haldane and Wiener. I now go on to consider two basic issues: 1), Why in a quantum universe having no events occurring outside human minds would different observers agree on what they see? and 2), Why in such a quantum universe would what they see be describable in classical terms? 


\section{Intersubjective Agreement}

Within the framework of the quantum mechanical picture of the universe described above, let us consider the possibility that the events occur only in conjunction with projection operators $P$ that act nontrivially (i.e., as something other than a unit operator) only on systems confined to human brains, or similar organs. In particular, let us suppose that no collapse of a wave function occurs in connection with a mechanical measuring device. In this situation the question arises: why do different observers normally agree on what they see; e.g., why do they all agree that the pointer on a measuring device that they all are observing has swing, say, to the left, and not to the right?

To discuss this question it is enough to consider just two such observers, and to relace the state $|\Psi\rangle$ discussed earlier by a state of the form

$$
\begin{aligned}
\mid \Psi> & =a\left|\psi_{11}>\otimes\right| \psi_{21}>\otimes \mid \psi_{3 a 1}> \\
& \otimes\left[e\left|\psi_{4 a 1 x}>+f\right| \psi_{4 a 1 y}>\right] \\
& \otimes \mid \psi_{3 b 1}> \\
& \otimes\left[g\left|\psi_{4 b 1 z}>+h\right| \psi_{4 b 1 w}>\right] \\
& +b\left|\psi_{12}>\otimes\right| \psi_{22}>\otimes \mid \psi_{3 a 2}> \\
& \otimes\left[p\left|\psi_{4 a 2 u}>+q\right| \psi_{4 a 2 v}>\right] \\
& \otimes \mid \psi_{3 b 2}> \\
& \otimes\left[r\left|\psi_{4 b 2 c}>+s\right| \psi_{4 b 2 d}>\right]
\end{aligned}
$$

Here $\left|\psi_{11}\right\rangle$ and $\left|\psi_{12}\right\rangle$ are, as before, the two pertinent states of the atom; $\mid \psi_{21}>$ and $\left|\psi_{22}\right\rangle$ are the two corresponding states of the measuring device (e.g., $\mid \psi_{21}>\sim$ the pointer has swung to the left: $\mid \psi_{22}>\sim$ the pointer has swung to the right); $\mid \psi_{3 a j}>$ and $\mid \psi_{3 b j}>$ are the states associated with the early (unconscious) processing parts of the nervous systems of the observers " $a$ " and "b" having registered $\psi_{2 j}$, for $j=1$ or 2 .

The states $\left|\psi_{4 a 1 x}\right\rangle$ and $\left|\psi_{4 a 1 y}\right\rangle$ are two alternative possible brain correlates that have arisen in the brain of observer " $a$ " from the lower-level state $\left|\psi_{3 a 1}\right\rangle$. The doubling of the possibilities arises from the indeterminacy associated with quantum processes occurring in the brain of observer "a". Such an 
indeterminacy arises, for example, from quantum processes in the synapses in his brain. ${ }^{11}$ Actually, there will be many more than just two such possibilities, but two is enough to illustrate the point. The other states $\left|\psi_{4 a j k}\right\rangle$ and $\left|\psi_{4 b j \ell}\right\rangle$ are analogous brain correlates of thoughts for observers " $a$ " and " $b$ ", respectively.

Suppose observer " $a$ " has the experience correlated to the brain state $\mid \psi_{4 a 1 x}>$. This experience corresponds to the jump of the state $|\Psi\rangle$ to the state

$$
\left|\Psi^{\prime}>=N P_{4 a 1 x}\right| \Psi>
$$

where $N$ is the normalization factor that makes

$$
<\Psi^{\prime}\left|\Psi^{\prime}\right\rangle=1
$$

and

$$
\begin{aligned}
P_{4 a 1 x} & =\left|\psi_{4 a 1 x}><\psi_{4 a 1 x}\right| \\
& \otimes \prod_{s \neq 4 a} I_{s}
\end{aligned}
$$

where $s$ runs over the set of systems $\{1,2,3,4 a, 4 b)\}$, and $I_{s}$ is the unit operator in the Hilbert space corresponding to system $s$.

The states $\psi_{4 a j k}$ for $(j, k) \neq(1, x)$ should be orthogonal to $\psi_{4 a 1 x}$, because under this condition $\psi_{4 a j k}$ and $\psi_{4 a 1 x}$ are the brain correlates of definitely distinguishable experiences:

$$
<\psi_{4 a j k} \mid \psi_{4 a 1 x}>=\delta_{j 1} \delta_{k x}
$$

More generally,

$$
<\psi_{m c i k} \mid \psi_{n d j \ell}>=\delta_{m n} \delta_{c d} \delta_{i j} \delta_{k \ell}
$$

But then the conditions (2.1) through (2.6) imply that the state $\left|\Psi^{\prime}\right\rangle$, which is the state that exists just after the occurrence of the experiential event of observer " $a$ " that is correlated to $\left|\psi_{4 a x 1}\right\rangle$, is

$$
\begin{aligned}
\mid \Psi^{\prime}> & =\left|\psi_{11}>\otimes\right| \psi_{21}>\otimes\left|\psi_{3 a 1}>\otimes\right| \psi_{4 a 1 x}> \\
& \otimes \mid \psi_{3 b 1}> \\
& \otimes\left[g\left|\psi_{4 b 1 z}>+h\right| \psi_{4 b 1 w}>\right] .
\end{aligned}
$$

At this stage of the sequential process of actualization no selection has yet been made between the two states $\left|\psi_{4 b 1 z}\right\rangle$ and $\left|\psi_{4 b 1 w}\right\rangle$ : i.e., observer "b" has 
not yet had his experience pertaining to the position of the pointer. But both of the possibilities available to him, namely $\left|\psi_{4 b 1 z}\right\rangle$ and $\left|\psi_{4 b 1 w}\right\rangle$, have $j=1$, and hence correspond to his seeing the pointer in the position specified by $j=1$ : both possibilities correspond to his seeing the pointer swung to the left. Thus both observers will agree that the pointer has swung to the left: intersubjective agreement is automatically assured by the quantum formalism.

According to the basic postulate (1.2), the probability for this event correlated to $\mid \psi_{4 a 1 x}>$ to occur is $|a|^{2}|e|^{2}$. If, contrary to the supposition made at the beginning of this section, there had been a prior event associated with the action of the device (i.e., a projection onto $P_{21} \mid \Psi>$ )) then, according to (1.2), the probability for this prior event to occur would have been $|a|^{2}$. Under the condition that this prior event did occur, the probability for the occurrence of the subsequent experiential event correlated to $\mid \psi_{4 a 1 x}>$ would be $|e|^{2}$. Thus the probability for this final event to accur is $|a|^{2}|e|^{2}$ in both cases: the probability for the occurrence of the experiential event does not depend upon whether the prior event at the level of the device occurred or not! Thus there is, in this example, (as in general) no empirical evidence to support the idea that an event occurs at the level of the device.

If we assume, in spite of this complete lack of any supporting evidence, that an event at the level of the device does in fact occur then the question arises: why does the jump take the device either to the state $\left|\psi_{21}\right\rangle$ or to the state $\left|\psi_{22}\right\rangle$, rather than to some linear combination of them? Why should the classically describable states $\left|\psi_{21}\right\rangle$ and $\left|\psi_{22}\right\rangle$ be singled out at the level of the quantum mechanical device itself, before any involvement or interaction with a potential human observer has occurred.

Of course, one can permit this prior event to occur without altering the probabilities associated with our experiences. Hence at some practical level one may wish to assume, or pretend, that this event at the level the device does occur. But in a realistic context, as opposed to a pragmatic one, this fact that this extra jump could occur without altering the propensities pertaining to our human experiences does not seem to be a sufficient reason for Nature to make this jump. If Nature should, nevertheless, choose to make a jump at the level of the device then why should she choose to actualize just a single one of the classically describable states, $\left|\psi_{21}\right\rangle$ or $\left|\psi_{22}\right\rangle$, rather than some 
linear superposition of them? Jumps to such superpositions would, to be sure, alter the empirically validated predictions of quantum theory. Hence we know empirically that jumps to such linear combinations do not occur. But in a realistic setting there should be a general physical principle that dictates which kind of states are actualized by the quantum jumps, and the fact that we cannot, in practice, detect the occurrence of certain kinds events is not a satisfactory general principle: it is based practical considerations rather than basic structure, and is too anthropocentric.

Because events occurring at the level of the devices must have a classical character that is hard to explain within a naturalistic framework, and because there is absolutely no empirical evidence to support the idea that events occur at this level, we are led to examine the more parsimonious assumption that the quantum events or jumps (i.e., the abrupt reductions of the quantum states) are associated primarily only with more complex systems, such as brains and similar organs: such jumps, by themselves, are sufficient to explain all of the scientifically accepted empirical evidence available to us today. But then a similar question arises: why, in a realistic framework, should the brain events associated with the perceptions of external objects correspond to experiences of objects that are classically describable if these objects themselves, before they are perceived, are represented by superpositions of such states? That is, although we know, on empirical grounds, within the framework of our theory, and to the extent that the structure of each experience mirrors ${ }^{12}$ the structure of its brain correlate, that the events at the level of brain correlates of perceptions must actualize brain states that have classically describable aspects, nevertheless the question arises: why should classical conditions be singled out in this way within a quantum universe? Is there something intrinsically classical about the character of possible perceptions; something that then forces any brain correlate that mirrors one of these perceptions to have corresponding classical aspects? That is, must we resort at this stage to some essentially metaphysical reason? Or, on the contrary, can the classical character of the brain events, and hence of mirroring thoughts, be deduced from strictly physical consideration alone? 


\section{Consciousness and Survival}

William James observed that "the study of the phenomena of consciousness which we shall make throughout this book shows us that consciousness is at all times primarily a selecting agency." ${ }^{13}$ Note that this conclusion is based on a survey of phenomena, rather than on our immediate subjective feelings.

Our most important and rudimentary choices, such as fight or flight, have to do with our survival. Thus from a naturalistic, or purely physical, point of view the character of consciousness ought to be a consequence of evolutionary pressures.

Within the framework of classical mechanics no such connection is possible, for in that framework the entire course of natural history is completely fixed by microscopic considerations involving only particles and local fields. Any additional structures that we might care to identify, as "realities" are, insofar as they are efficacious, completely reducible to these microscopic ones, and hence are, as far as the dynamical development of any system is concerned, completely gratuitous: how they are constructed from, or are related to, the elementary microscopic realities, or whether they exist at all, has no bearing on the survival of any organism. But within the framework of quantum mechanics developed here consciousness does have a causally efficacious role that is tied directly to the selections of courses of action: consciousness is a bone fide selecting agency. Thus it becomes at least logically possible within the quantum framework to link the character of human consciousness to the evolutionary pressures for human survival.

Considerations of wholeness led Haldane to suggest that mind is linked to resonance phenomenoma. This intuition has been revived by Crick and $\mathrm{Koch}^{14}$, who suggest that the empirically observed ${ }^{15} 40$ Hertz frequencies that lock together electrical activities in widely separated parts of the brain is associated with consciousness. I shall accept this general idea of a resonance type of activity involving widely separated parts of the brain as a characteristic of the brain correlate of a conscious thought, although the idea of an "attractor" would do just as well. Since energy is available in the brain, the feed-back resonance of a public address system is a suitable metaphor. ${ }^{16}$

Generally a superposition several alternative possible "resonant" or "at- 
tractor" states will emerge from the quantum dynamics. ${ }^{11,12}$.This is illustrated by the different states $\psi_{4 a 1 x}$ and $\psi_{4 a 1 y}$ in (2.1). These alternative possible states have certain "classical" aspects: riding on a chaotic ocean of microscopic activity there will be certain collective variables that are relatively stable and slowly changing, and that can be called the macroscopic variables of the system. They will be the variables of classical electromagnetism: charge densities, electric field strengths, etc., and they are defined by averaging over regions that are small compared to the brain, but large compared to atoms. The states $\psi_{4 a 1 x}$ and $\psi_{4 a 1 y}$, or, more accurately, the projection operators $P_{[4 a 1 x]}$ and $P_{[4 a 1 y]}$ corresponding to collections of many micro states subsumed under the macroscopic characteristics identified by the symbols [4a1x] and [4a1y], will be characterized in terms of these macroscopic (classical) variables. These macro-variables will contain both the information pertaining to the location of the pointer on the external device (specified here by $j=1$ ), and also the additional macroscopic specifications labelled by the indices $x$ and $y$. Notice that a sum $P$ of orthogonal projection operators $P_{i}$,

$$
P=\sum_{i} P_{i} \quad P_{i} P_{j}=\delta_{i j} P_{i}
$$

is a projection operator: $P^{2}=P$. Hence the quantum rules described above apply to these operators $P_{[4 a 1 x]}$ and $P_{[4 a 2 y]}$ that are formed as sums over sets of orthogonal operators $P_{i}$ that meet the indicated specifications.

Two questions now arise. The first is this: why should evolutionary pressures tend to force the events in brains to correspond to projection operators $P$ that project onto "resonance" or "attractor" states that involve large parts of the brain, and many neurons, rather than, say, to projection operators that project onto macroscopically specified states of individual neurons?

The second question is this: why, if the evolutionary pressures do tend to force the brain events to correspond to large structures, such as large-scale resonances or attractors, do they not tend to force the events even further in the direction of largeness, and allow them to correspond to superpositions of classically describable macroscopically specified states, instead of individual ones.

These two questions are addressed in the following two sections. 


\section{Survival Advantage of Having Only Top-Level Events.}

A principal task of the brain is to form templates for possible impending actions. Each such template is conceived here to be resonance or attractor state that involves activity that is spread out over a large part of the brain. The evolutionary pressure for survival should tend to promote the emergence of a brain dynamics that will produce the rapid formation of such top-level states. However, as will be discussed in this section, the occurrence of quantum events at lower levels (e.g., at the levels of individual neurons, or smaller structures) will act as a source of noise that will tend to inhibit the maximally efficient formation of these top-level states. Thus the evolutionary pressure for survival will tend to force the events in brains to occur preferentially at the higher level, i.e., to actualize mainly the top-level states. Each of our conscious thoughts seems to have only the information that is present in the part of the brain state that is actualized by one of these top-level events. ${ }^{12}$ Hence it is natural to postulate ${ }^{12}$ that the top-level states actualized by quantum events are precisely the brain correlates of our conscious thoughts.

In the simple example examined earlier there was a separation at each of the $N-1$ macroscopic levels into two macroscopically distinct branches, labelled by $j=1$ or 2 , and there was consequently a natural way to define the projection operators $P_{n 1}$ and $P_{n 2}$ at the lower levels that were effectively equivalent, within that measurement context, to the two final projection operators $P_{N 1}$ and $P_{N 2}$ that were directly associated with the two distinct classically describable experiences. However, if we try to trace back through the brain dynamics to find the lower-level projection operators that are equivalent to the ones associated with top-level events then we would find operators that are neither simple nor natural. Moreover, there would be no rationale for projecting at some lower level onto precisely the low-level brain states that would eventually lead to the various distinct top-level states.

There is, on the other hand, a widely held notion that brain activity is basically classical at the level of neuron firings, so that there never is a superposition of, for example, a state in which some neuron is firing and a state in which it is not firing.

To reconcile this intuitive idea with our realistically formulated quantum 
mechanics we would need to have low-level events that would prevent quantum superpositions of distinct classically describable states of individual neurons from developing, or persisting. There is, however, a problem in implementating this idea. The processes occurring in brains depend upon the probability densities for various atoms and ions to be in various places at various times. These densities are essentially continuous in quantum theory, and this makes the brain dynamics essentially continuous: a neuron can fire a little sooner, or a little later, or a little more strongly or weakly, etc. The quantum propensities define, therefore, only an amorphous structure, insofar as no events occur. But then the question is: how, in this initially amorphous situation, does one introduce a set of events (quantum jumps or collapses) that will keep the lower level (i.e., neuronal) situation essentially classical? How does one characterize the appropriate low-level projection operators $P_{i}$ onto classical states in cases where the quantum dynamics itself does not separate the state into classically distinct and non overlapping low-level branches? The "measurement" situation discussed earlier is essentially misleading, if applied to the present case, because it did not involve this problem of reducing an amorphous quantum state that is not already decomposed into well separated "classical" parts into a description that is essentially classical.

A way of dealing with this problem was proposed in ref. 17. It is based on coherent states. ${ }^{18,19}$ For any complex number $z=(q+i p) / \sqrt{2}$ let $|z\rangle$ define a state whose wave function in (a one-dimensional) coordinate space is

$$
\psi_{z}(x)=\langle x| z>=\pi^{-1 / 4} e^{i p x} e^{-\frac{1}{2}(x-q)^{2}} .
$$

This state is normalized,

$$
<z\left|z>=\sum_{x}<z\right| x><x \mid z>=1,
$$

and it satisfies the important property ,

$$
\begin{aligned}
& \int \frac{d z}{\pi}|z><z| \equiv \int \frac{d p d q}{2 \pi}|z><z| \\
& \equiv \sum_{z}|z><z| \equiv \sum_{z} P_{z}=I
\end{aligned}
$$

where " $I$ " is again the identity or unit operator. 
A transformation that takes a density operator $\rho$ that describes a slowly varying state into an "equivalent" statistical mixture of "classical" states $\mid z>$ is

$$
\rho \rightarrow \rho^{\prime}=\sum_{z} P_{z} \rho P_{z} .
$$

This mixture $\rho^{\prime}$ is "equivalent" to $\rho$ in the sense that if $\left\langle x|\rho| x^{\prime}\right\rangle$ is a slowly varying function of its two variables $x$ and $x^{\prime}$, on the scale of the unit interval that characterizes the width of the "classical" states $|z\rangle$, then, for any $z^{\prime}$, one has

$$
<z^{\prime}|\rho| z^{\prime}>\approx<z^{\prime}\left|\rho^{\prime}\right| z^{\prime}>
$$

Proof:

$$
\begin{aligned}
& \left.<z^{\prime}\left|\rho^{\prime}\right| z^{\prime}\right\rangle \\
& =\sum_{z}<z^{\prime}|z><z| \rho|z><z| z^{\prime}> \\
& \approx \sum_{z}<z^{\prime}\left|z><z^{\prime}\right| \rho\left|z^{\prime}><z\right| z^{\prime}> \\
& \left.=<z^{\prime}|\rho| z^{\prime}\right\rangle
\end{aligned}
$$

where the fact that $\left\langle z^{\prime} \mid z\right\rangle$ is strongly peaked at $z^{\prime}=z$ is used. Thus the transformation from $\rho$ to $\rho^{\prime}$ leaves the diagonal (and the nearly diagonal) elements of $\left\langle x|\rho| x^{\prime}\right\rangle$ approximately unchanged, but changes $\rho$ to a classically interpretable mixture of states that are localized in coordinate space, on a certain (unit) scale.

The relationship

$$
\operatorname{Tr} \rho^{\prime}=\operatorname{Tr} \rho
$$

also hold.

Proof:

$$
\begin{aligned}
& \sum_{x}<x\left|\rho^{\prime}\right| x> \\
& =\sum_{x} \sum_{z}<x|z><z| \rho|z><z| x> \\
& =\sum_{z}<z|\rho| z>\sum_{x}<z|x><x| z>
\end{aligned}
$$




$$
\begin{aligned}
& =\sum_{z} \sum_{x} \sum_{x^{\prime}}<z|x><x| \rho\left|x^{\prime}><x^{\prime}\right| z> \\
& =\sum_{x} \sum_{x^{\prime}}<x|\rho| x^{\prime}><x^{\prime}|x\rangle \\
& \left.=\sum_{x}<x|\rho| x\right\rangle .
\end{aligned}
$$

Suppose the dynamics is such as to generate and sustain a state $\mid 0>$ (i.e., $\mid z=0>$ ) that is a component of a top-level resonant state. The property of the dynamics to sustain the state $\mid 0>$, but to cause states orthogonal to it to dissipate, is expressed by the conditions

$$
U(t) \mid 0>=10>
$$

for all $t>0$, where $U(t)$ is the unitary operator that generates the evolution from time zero to time $t$, and for each pair $\left(z^{\prime}, z\right)$

$$
<z^{\prime}\left|U(t)\left(I-P_{0}\right)\right| z>\Longrightarrow 0
$$

where $P_{0}=|0><0|$, and the double arrow signifies the large-time limit. Then for any pair $\left(z^{\prime}, z^{\prime \prime}\right)$ we have, by virtue of (4.8) and (4.7), (and assuming that $<z \mid \rho$ and $\rho \mid z>$ tend to zero for large $|z|)$,

$$
\begin{aligned}
& <z^{\prime}\left|U(t) \rho U^{\dagger}(t)\right| z^{\prime \prime}> \\
\Longrightarrow & <z^{\prime}\left|P_{0} \rho P_{0}\right| z^{\prime \prime}> \\
= & <z^{\prime}|0><0| \rho|0><0| z^{\prime \prime}>
\end{aligned}
$$

Similarly, for any pair $\left(z^{\prime}, z^{\prime \prime}\right)$ and slowly varying $\rho$,

$$
\begin{gathered}
<z^{\prime}\left|U(t) \rho^{\prime} U^{\dagger}(t)\right| z^{\prime \prime}> \\
=\sum_{z}<z^{\prime}|U(t)| z><z|\rho| z><z\left|U^{\dagger}(t)\right| z^{\prime \prime}> \\
\Longrightarrow \sum_{z}<z^{\prime}|0><0| z><z|\rho| z><z|0><0| z^{\prime \prime}> \\
\approx \sum_{z}<z^{\prime}|0><0| z><0|\rho| 0><z|0><0| z^{\prime \prime}> \\
=<z^{\prime}|0><0| \rho|0><0| z^{\prime \prime}>
\end{gathered}
$$


Thus the change from $\rho$ to $\rho^{\prime}$ makes little difference in these matrix elements: the statistical mixture of classical states $\rho^{\prime}$ have approximately the same matrix elements as the original $\rho$.

After some finite time, however, an originally smooth $\rho$ will, by virtue of (4.7) and (4.8), develop a classical component proportional to $|0\rangle\langle 0|=P_{0}$ that will stand out from the smooth background. Consider, therefore, the effect of the dynamics on $\rho$ and $\rho^{\prime}$ for this part of $\rho$ proportional to $\rho_{0}=P_{0}$ :

$$
\begin{gathered}
<z^{\prime}\left|U(t) \rho_{0} U^{\dagger}(t)\right| z^{\prime \prime}> \\
\Longrightarrow<z^{\prime}|0><0| z^{\prime \prime}>,
\end{gathered}
$$

whereas

$$
\begin{gathered}
<z^{\prime}\left|U(t) \rho_{0}^{\prime} U^{\dagger}(t)\right| z^{\prime \prime}> \\
=\sum_{z}<z^{\prime}|U(t)| z><z \mid 0> \\
\times<0|z><z| U^{\dagger}(t) \mid z^{\prime \prime}> \\
\Longrightarrow \sum_{z}<z^{\prime}|0><0| z><z|0><0| z><z|0><0| z^{\prime \prime}> \\
=<z^{\prime}|0><0| z^{\prime \prime}>\times \sum_{z}(<0|z><z| 0>)^{2} .
\end{gathered}
$$

But

$$
0<(<0|z><z| 0>)<1 \text { for all } z \neq 0
$$

and

$$
\sum_{z}<0|z><z| 0>=1
$$

Hence

$$
\sum_{z}(<0|z><z| 0>)^{2}<1 .
$$

Thus the effect of introducing the events that convert $\rho$ to the classical approximation $\rho^{\prime}$ has the effect of disrupting the preservation of the state $\mid 0>$ : the probability of staying in this "preferred" state is diminished by the effects of introducing the low-level events. 
Although this result was obtained under simplifying assumptions that allowed us easily to compute the effect, the conclusion is general. It arises essentially from the fact that the transformation $\rho \rightarrow \rho^{\prime}$ "flattens out" a bump in $\rho$ that is already of a classical size, and hence inhibits the emergence of a single classical state from an amorphous background.

The problem, in the general context, is this: the quantum dynamics can be such that certain resonance states (preferred for their survival advantages) will emerge from an amorphous backgrounds of quantum probabilities. (See ref. 16). Each of these resonance states will be a collective phenomena involving many neurons. The emerging resonant state will be characterized by specific relationships in the timing of the firings of the various neurons. The incipient resonances can generate bumps, but it is not known to the system beforehand which specific combinations of firing timings will eventually emerge from the smooth quantum soup via the complex feed-back mechanisms.

The quantum dynamics allows such self-generating states to emerge from the amorphous quantum soup with a certain maximal efficiency, because all of the possible overlapping configurations of classical possibilities are simultaneously present, and their consequences are simultaneously explored by the quantum dynamics. After the dynamics has generated an output consisting of a superposition of distinct classical top-level resonating states then an event can occur that will select one of these top-level possibilities without interfering with the dynamics that has just generated the various top-level possibilities. But if events are required to occur at a lower level, in order to impose the condition of classical describability there, then, in order to maintain the maximal efficiencies for the production of the top-level states, these events would have to project upon states that have optimal relationships among the timings of the firings of the neurons. But these timings are not yet known to the system. The introduction of a statistically distributed set of low-level events can achieve the demanded reduction to a classical description at the low level, as in our example, but this disruption of the quantum dynamics will undoubtedly, just as it did in our example, inject into the evolution of the system an element of noise that will tend to reduce the efficiency of generating and sustaining the top-level states.

Physicists have, today, no idea of what, if any, property of a system determines the level at which the "events" associated with this system occur. But 
within a naturalistic setting this level should be determined by some characteristic of that system itself. If this is true, then the arguments given above would lead to the conclusion that evolutionary pressures should cause brains to evolve in such a way as to shift the events occurring in alert brains to the top level, thus leaving the dynamics at the neuronal level and below controlled by the local deterministic quantum law of evolution, namely the Schroedinger equation. This resolves the logical problem of how to tie the description of the behavior of the neurons in a rational way to the description of the intertwined chemical processes that are so crucial to their functioning. The solution: use the quantum description throughout, making full use, of course, of the approximate validity of classical concepts entailed by the decoherence-type effects illustrated in equation (5.4) below. 


\section{Classical Description}

Classical concepts have entered in an important way into the above description of the process of actualization of the quantum states: the projection operators associated with the events have been characterized by classically describable conditions on certain macroscopic variables. The question thus arises: why should classical concepts enter at all into the evolution of the quantum universe? Why should the quantum events project onto states in which the values of macroscopic field variables at spacetime points are confined to small domains, instead of projecting onto superpositions of such classically describable states?

Here again an answer based on the survival of the species can be given. It is tied to the local character of the interaction and the concept of symbol.

A symbol is a physical structure that can be "interpreted" by a mechanism: the mechanism gives a characteristic response to the symbol. In our model the various actualized states in the brain, the brain correlates of thoughts, act as symbols. These states are characterized by definite values of macroscopic classical-type variables, and the motor responses are determined in large measure by classically describable reactions to the classically describable inputs provided by these symbols. But then the question is: why should the quantum events actualize states having this special classical character instead of superpositions of such states?

To find the answer suppose that the brain has evolved to a point where the brain correlates have been generated, and that for simplicity, these states are just two in number. Let these two brain correlates be denoted by $\left|\varphi_{1}\right\rangle$ and $\left|\varphi_{2}\right\rangle$. These two states are supposed to be characterized by macroscopic variables that are significantly different. Consequently, these two states will, because of the local character of the interaction, very quickly generate greatly differing (orthogonal) states in the embedding ocean of microscopic variables: the brain will, to a very good approximation, evolve to a state of the form

$$
|\psi>=a| \varphi_{1}>\left|\chi_{1}>+b\right| \varphi_{2}>\left|\chi_{2}\right\rangle,
$$

where the states $\left|\chi_{1}\right\rangle$ and $\left|\chi_{2}\right\rangle$ are orthogonal states in the imbedding space of microscopic degrees of freedom.

The importance of states such as (5.1) is that the significant information is concentrated into the classical level of description, i.e., in the states $\left|\dot{\varphi}_{1}\right\rangle$ 
and $\left|\varphi_{2}\right\rangle$, and this macroscopically represented information can control, in large measure, the ongoing evolution via the laws of classical physics. This provides the evident evolutionary advantage in having the events correspond to projection operators that act at the level of the macrovariables, for then the consequences of the selection associated with an event can be largely governed by deterministic classical laws. But the question before us now is whether there could be any additional advantage in having the events correspond to operators that project onto superpositions of such macrostates.

In the present simple example the question is whether it could be advantageous to have events that correspond to projection operator such as

$$
P=\left(c\left|\varphi_{1}>+d\right| \varphi_{2}>\right)\left(c^{*}<\varphi_{1}\left|+d^{*}<\varphi_{2}\right|\right) \times I_{\chi}
$$

with $c d \neq 0$.

The density operator in our example is

$$
\rho=|\psi><\psi|
$$

with $|\psi\rangle$ defined in (5.1). Our first observation is that

$$
\operatorname{Tr} P \rho=\operatorname{Tr} P \rho^{\prime},
$$

where

$$
\begin{aligned}
\rho^{\prime} & =|a|^{2}\left|\varphi_{1}>\right| \chi_{1}><\chi_{1}\left|<\varphi_{1}\right| \\
& +|b|^{2}\left|\varphi_{2}>\right| \chi_{2}><\chi_{2}\left|<\varphi_{2}\right| .
\end{aligned}
$$

Proof:

$$
\begin{aligned}
& \operatorname{TrP\rho } \\
& \begin{aligned}
=\sum_{x}< & x \mid\left(c\left|\varphi_{1}>+d\right| \varphi_{2}>\right)\left(c^{*}<\varphi_{1}\left|+d^{*}<\varphi_{2}\right|\right) \\
& \times\left(a\left|\varphi_{1}>\right| \chi_{1}>+b\left|\varphi_{2}>\right| \chi_{2}>\right) \\
& \times\left(a^{*}<\varphi_{1}\left|<\chi_{1}\right|+b^{*}<\varphi_{2}\left|<\chi_{2}\right|\right) \mid x> \\
=\left(c^{*}\right. & \left.<\varphi_{1}\left|+d^{*}<\varphi_{2}\right|\right) \\
& \times\left(a\left|\varphi_{1}>\right| \chi_{1}>+b\left|\varphi_{2}>\right| \chi_{2}>\right)
\end{aligned}
\end{aligned}
$$




$$
\begin{aligned}
& \times\left(a^{*}<\varphi_{1}\left|<\chi_{1}\right|+b^{*}<\varphi_{2}\left|<\chi_{2}\right|\right) \\
& \times\left(c\left|\varphi_{1}>+d\right| \varphi_{2}>\right) \\
= & c^{*} a a^{*} c+d^{*} b b^{*} d \\
= & |a|^{2}|c|^{2}+|b|^{2}|d|^{2} \\
= & T r P \rho^{\prime} .
\end{aligned}
$$

This means that the probability for the occurrence of the event associated with $P$ is the same for the density operator $\rho^{\prime}$ as it is for $\rho$.

Given the fact the information available for determining the subsequent (macroscopically controlled) dynamics is contained in $\rho^{\prime}$, what is the form of $P$ that least degrades this information? The answer is $P$ with $c d=0$ : the $P$ should be either $\left|\varphi_{1}><\varphi_{1}\right|$ or $\left|\varphi_{2}><\varphi_{2}\right|$.

For example, if $\left|\varphi_{1}\right\rangle$ corresponds to a very good choice for the organism, and $\mid \varphi_{2}>$ a very poor one, so that a well conditioned brain will give a $\rho$ with $|a|^{2} \simeq 1$ and $|b|^{2} \simeq 0$, and if the $P$ is given by (5.2) with $|c|^{2}=|d|^{2}=1 / 2$ then (5.6) shows that all the information about $|a|^{2}$ and $|b|^{2}$ will be lost: the result is $1 / 2\left(|a|^{2}+|b|^{2}\right)=1 / 2$ independently of $|a|^{2}$ and $|b|^{2}$. This special example already suggests the answer: $P$ should be either $\left|\varphi_{1}><\varphi_{1}\right|$ or $\left|\varphi_{2}><\varphi_{2}\right|$, in order to retain all the information. Any other choice causes a degradation of the information generated by the brain dynamics. In general, the optimal choice for the $P$ is that it should be one of a set of $P$ 's each of which projects onto a single one of the classically described states generated by the brain dynamics: otherwise some information generated by the brain will be lost, and the likelihood that the organism will survive will be diminished. 


\section{Inequivalence of Other Ontological Interpretations}

There is an alternative interpretation of quantum theory that can be construed as an ontology - i.e., as a putative description of nature herslf but in which there are no collapse events. This is Everett's "many-minds" interpretation. ${ }^{20}$ In this interpretation there is no natural place to introduce the mental events because nothing ever "happens": the entire course of history is continuously laid out on a spacetime plot, with no clear notion of any "actual happenings" or events.

It is difficult, and I think impossible, to give any rational meaning to "probability" in an Everett world where there are no definite happenings or events. Indeed, because the components of a superposition must be combined conjunctively - since in principle they can interfere with each other - each of the possibilities present in the evolving state of the universe must exist together with every other one. Hence they cannot have the independent probabilities for coming into existence that is allowed for the elements of a disjunctive combination of possibilities. Indeed, all of the branches of the state vector are supposed to exist in unison. The mere fact that that this physical state can be separated into a superposition of components that correspond to noncommunicating realms of experience, or to distinct recorded histories, does not, by itself, make the probabilities for the coming into existence of these various physical components any different from the single probability of the whole of which they are the simultaneously existing parts, or from the probabilities that these parts would have if the associated experiential realms were not completely noncommunicating. Yet, for empirical reasons, tiny probabilities must often be assigned to some branches and large probabilities to others, even though all of them exist in unison, according to the Everett view.

The only apparent rational way to reconcile these requirement is to introduce into the ontology some entities, besides the quantum state itself, for the probabilities to refer to. To make the necessary tie-in to empirical data these must correspond in some way to growing historical experiencable records that are allowed to prolong themselves into the future in alternative possible ways, with the alternative possibilities populating the different branches of the state vector of the evolving universe. Then the model becomes endowed with 'happenings', namely the selections or choices of the prolongations of each of these histories 
into the future, and, correspondingly, with choices between the simultaneously existing branches of the state vector.

The probabilities for these events are supposed to be governed by the quantum rules. However, in the Everett framework these events do not influence the evolution of the quantum state: the influence or control is unidirectional, from the quantum state to the events. Thus everything is controlled by the Schroedinger equation except for individual choices, which, however, are buried in a population whose statistical properties are controlled by the locally deterministic Schroedinger equation. Thus, within this framework, no arguments based on survival of organisms can be used to determine just where to locate the particular physical activities in our brains that correspond to our thoughts. Any placement would be equivalent, as far as survival is concerned, to any other one, because the placement is not connected to any difference in the dynamical evolution of the statistical ensemble that constitutes the full system: just as in classical mechanics, the evolution of the full system is completely deterministic, and is independent of where, in the dynamical unfolding, nature chooses to place the physical correlate of the epiphenomenal consciousness.

Likewise in Bohm's nonlocal deterministic ontological model ${ }^{21}$ the placement of the nonefficacious consciousness within the deterministically evolving universe has no effect upon the course of nature, and hence none upon the suvival of the species. Hence the mechanisms for the evolution of consciousness discussed here cannot be operative in either of these alternative frameworks, essentially because consciousness is not efficacious in these models 


\section{Conclusions}

It was suggested by Haldane and Weiner, shortly after the birth of quantum mechanics, that this profoundly deepened understanding of the nature of matter allows mind to be liberated from the epiphenomenal status assigned to it by classical mechanics, and to become, instead, an aspect of nature that is interactive with, rather than subservient to, the local deterministic matter-like aspect of nature that was mistakenly identified as the entire physical universe by classical mechanics. This suggestion of Haldane and Weiner remains viable today and, indeed, is being vigorously pursued. Haldane's further suggestion that mind is associated with a resonance phenomena has been revived by Crick and Koch, without its quantum foundation, and is the basis one of today's premier research programs on the mind-brain problem.

If the level of brain dynamics at which the quantum event occurs is determined by the physical characteristics of that organ itself, then there should exist effective evolutionary pressures that will tend to raise this level to the top level, which is characterized as the formation of macroscopic templates for possible impending action in which classically describable aspects, expressed in terms of the macroscopic variables of classical electrodynamics, form symbols for the activation of processes that, at least in the case of motor processes, remain largely controlled by macroscopic variables acting in accordance with classical laws. The general brain process will remain essentially quantum mechanical. On the other hand, due to the local character of the interaction, there will also be evolutionary pressure for the top-level event not to go beyond the classically describable level to the level of superpositions of classically describable states. Thus the classical character of our thoughts, if assumed to mirror the relational structures specified by the projection operators $P$ associated with the corresponding brain events ${ }^{12}$, can be naturally explained within the mathematical framework of quantum mechanics.

This evolution-based explanation of the classical character of our thoughts, and hence of the observed physical world itself, is independent of whether or not classically describable events occur at the level of mechanical measuring devices.

Although the argument given above was specialized to the human organism, it applies equally well to all organisms whose structure is governed by 
evolutionary pressures for survival: the general conclusion would be that in all such organisms the freedom that inheres in each of its component subsystems to make quantum choices should be suppressed to the extent that such choices interfere with the quantum process of the organism as a whole to create top-level templates for possible actions, and that there should be in all such organisms top-level events each of which actualizes one of the templates for possible action generated by the local-deterministic part of the quantum dynamical process. The way in which the selected event is singled out from all the other possibilities generated by the quantum dynamics is not yet a part of what science has revealed to us. 


\section{References}

1. J.B.S. Haldane (1934); Philos. Sci. 1, 78-98.

2. N. Wiener (193?); Philos. Sci. 3, 307-319.

3. D. Bohm (19??); in Norbert Wiener: Collected Works Vol.IV p.97

4. N. Bohr (1934); Atomic Theory and the Description of Nature (Cambridge University Press, Cambridge) p.18

5. N. Bohr (1963); Essays 1958/1962 on Atomic Physics and Human Knowledge (Wiley, New York) p.60 [See also ref.12 p.62].

6. A. Einstein (1949); in Albert Einstein: Philosopher-Scientist ed. P.A. Schilpp (Tudor, New York) p.81.

7. A. Einstein ibid p.87.

8. N. Bohr (1958); Atomic Physics and Human Knowledge (Wiley, New York) p.20.

9. J. von Neumann (1932); Mathematical Foundations of Quantum Mechanics (Princeton University Press, Princeton, 1955) (English translation).

10. N. Bohr (1934) ibid p.75.

11. H.P. Stapp (1991); Found. Phys. 21, 1451-1477.

12. H.P. Stapp (1993); Mind, Matter, and Quantum Mechanics (Springer Verlag, Berlin Heidelberg New York London Paris Tokyo Hong Kong Barcelona Budapest) Ch. 6 .

13. W. James (1890); The Principles of Psychology (Dover, New York) Vol I, p.XX.

14. F. Crick (1994); The Astonishing Hypothesis: The Scientific Search for the Soul (Scribner, New York).

15. A.K. Engle, A.K. Kreiter, P. Koenig, and W. Singer, Proc. Nat. Acad. Sci. USA 88, 6048-6052. 
16. H.P. Stapp (1995); in Fundamental Problems in Quantum Theory (New York Academy of Science, New York).

17. H.P. Stapp (1987); in Quantum Implications: Essays in Honor of David Bohm (Routledge and Paul Kegan, London and New York).

18. J.R. Klauder and B. Skagerstam (1985); Coherent States (World Scientific, Singapore).

19. J.R. Klauder and E.C.G. Sudarshan (1968); Fundamentals of Quantum Optics (W.A. Benjamin, New York).

20. Hugh Everett III (1957); Rev. Mod. Phys. 29, 454-62: H.P. Stapp (1980); Found. Phys. 10, 767-795.

21. D. Bohm and B. Hiley (1993); The Undivided Universe: An Ontological Interpretation of Quantum Theory (Routledge, London and New York). 